\title{
X-ray speckle contrast variation at sample-specific absorption edges
}

\author{
Cornelia C. Retsch, Yuxin Wang, Sean P. Frigo, G. Brian Stephenson, Ian McNulty \\ Argonne National Laboratory \\ 9700 South Cass Avenue \\ Argonne, 1160439 \\ retsch@aps.anl.gov
}

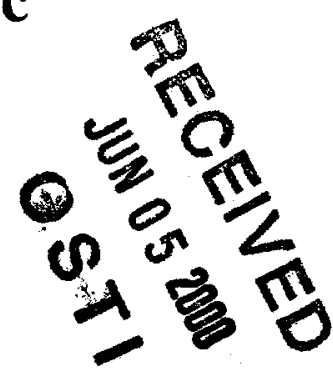

\begin{abstract}
We measured static x-ray speckle contrast variation with the incident photon energy across sample-specific absorption edges. We propose that the variation depends strongly on the spectral response function of the monochromator.

(01999 Optical Society of America

OCIS codes: (000.2170) Equipment and techniques; (030.6140) Speckle; (340.6720) Synchrotron radiation
\end{abstract}

\section{Introduction}

Speckle techniques have been introduced to the $x$-ray regime during recent years [e.g., 1-3]. Most of these experiments, however, were done at photon energies above $5 \mathrm{keV}$. We are working on this technique in the 1 to 4 $\mathrm{keV}$ range, an energy range that includes many important $\mathrm{X}$-ray absorption edges, e.g., in $\mathrm{Al}, \mathrm{Si}, \mathrm{P}, \mathrm{S}$, the rare-earths, and others. To our knowledge, the effect of absorption edges on speckle contrast has not yet been studied. In this paper, we present our initial measurements and understanding of the observed phenomena.

\section{Theory}

For analyzing speckle patterns, a very useful tool is the normalized, baseline-subtracted intensity autocorrelation function [4]. In the nonmonochromatic case, this function depends on the monochromator spectral response function, as well as the intensity $\langle I(k)\rangle$ in the speckle pattern at wave number $k$, averaged over the position in the detector plane, and the mutual intensity function of the system. The width of this function describes the average width of a speckle, and its height is a good measure of the contrast in the speckle pattern. The contrast can depend strongly on the monochromaticity of the sample illumination [5].

If $\langle I(k)\rangle$ does not vary significantly over the width of the spectral response function, it can be treated as a constant factor [4]. The intensity autocorrelation function can then be solved analytically for a Gaussian spectral response function [4,5]. This solution does not account for any effects of an absorption edge on the contrast.

\section{Experimental Results}

We measured the variation in speckle contrast at two sample-specific absorption edges at the SRI-CAT beamline 2-ID-B [6,7] at the Advanced Photon Source. The two different samples we used were aluminum and zinc powder (Al K-edge: 1.56 keV; $\mathrm{Zn}$ L-edge: $1.02 \mathrm{keV}$ ). The aluminum powder consisted of 1 to $3 \mu \mathrm{m}$-sized grains, the zinc powder consisted of submicrometer-sized grains. The experimental setup is shown in Figure 1.

The disordered powder samples were illuminated with a nearly coherent $\mathrm{x}$-ray beam of $5-\mu \mathrm{m}$ diameter selected by a pinhole. The degree of coherence was higher than $90 \%$. A guard slit in front of the sample blocked parasitic scattering from the pinhole. The speckle patterns were recorded with a directly backside-illuminated charge-coupled device camera at $500 \mathrm{~mm}$ distance from the samples as the incident photon energy was varied across the absorption edges. Examples of the recorded speckle patterns below the edge and right at the edge are shown in Figure 2. The size of the shown area extends in reciprocal space ( $q$-space) from $\sim 1.0 \times 10^{-3} \AA^{-1}$ to $\sim 10.4 \times 10^{-3} \AA^{-1}$ in the horizontal and from $\sim-4.7 \times 10^{-3} \AA^{-1}$ to $\sim 4.7 \times 10^{-3} \AA^{-1}$ in the vertical direction, which corresponds to length scales in the sample of the order of $0.6 \mu \mathrm{m}$ to $6 \mu \mathrm{m}$. 


\section{DISCLAIMER}

This report was prepared as an account of work sponsored by an agency of the United States Government. Neither the United States Government nor any agency thereof, nor any of their employees, make any warranty, express or implied, or assumes any legal liability or responsibility for the accuracy, completeness, or usefulness of any information, apparatus, product, or process disclosed, or represents that its use would not infringe privately owned rights. Reference herein to any specific commercial product, process, or service by trade name, trademark, manufacturer, or otherwise does not necessarily constitute or imply its endorsement, recommendation, or favoring by the United States Government or any agency thereof. The views and opinions of authors expressed herein do not necessarily state or reflect those of the United States Government or any agency thereof. 


\section{DISCLAIMER}

Portions of this document may be illegible in electronic image products. Images are produced from the best available original document. 


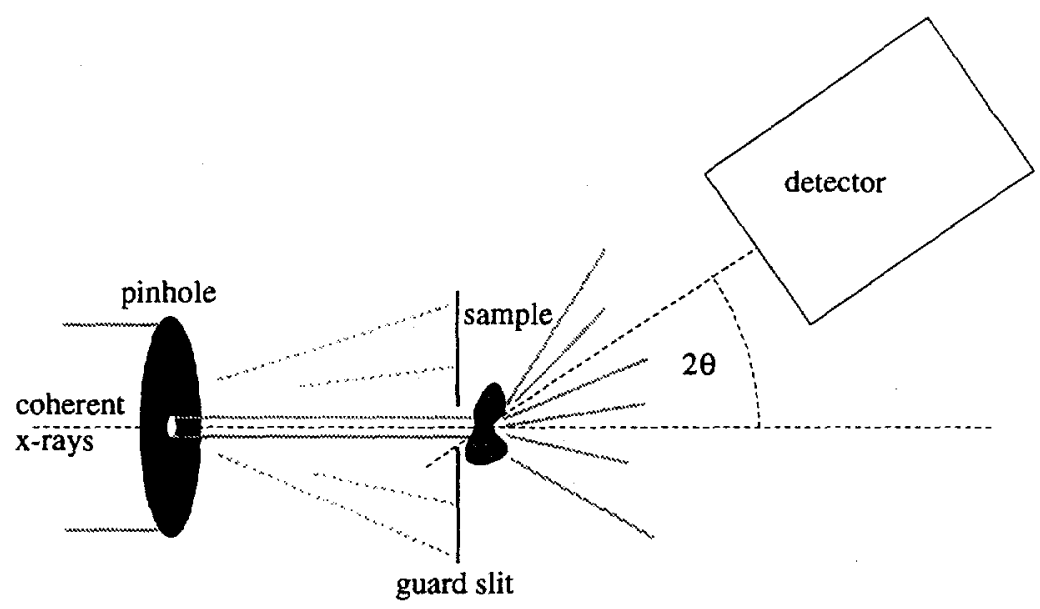

Fig. 1. Experimental setup.
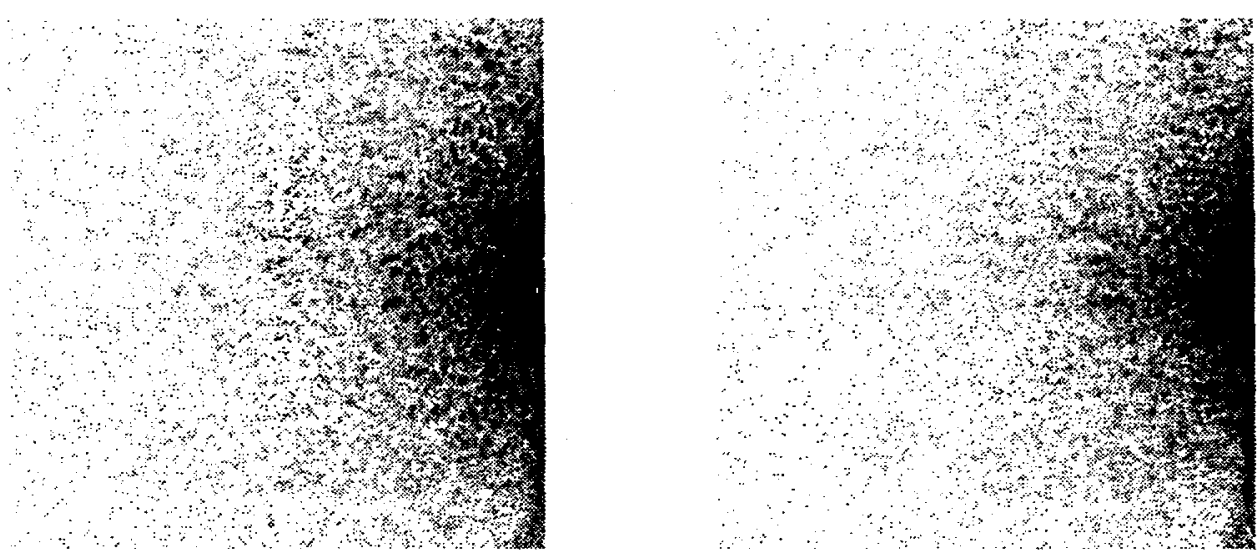

Fig. 2. Speckle patterns from the aluminum powder sample below the absorption edge (left) and at the absorption edge (right). The area shown is a square of $9.4 \times 10^{-3} \AA^{-1}$ lengths in $q$-space.

We retrieved the speckle contrast from the data by calculating the normalized and background-subtracted intensity autocorrelation function of the speckle pattern. The values at different positions in $q$-space between $q=1 \times 10^{-3} \AA^{-1}$ and $q=5.5 \times 10^{-3} \AA^{-1}$ were averaged to show the overall trend of the behavior; any $q$-dependence is reflected in the error bars. Figure 3 shows the results. The measured contrast decreases at the absorption edge and slowly increases towards its previous level afterwards. This effect is not due to the overall loss in intensity at the edge as the measured intensity has been normalized prior to contrast analysis.
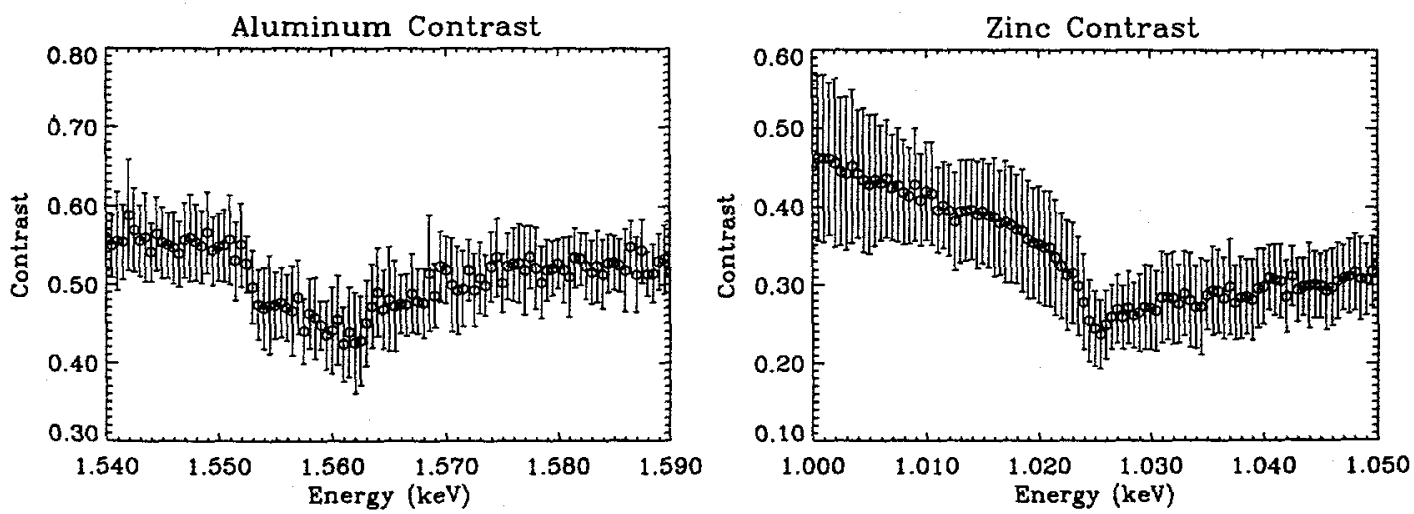

Fig. 3. Speckle contrast across the sample-specific absorption edge for aluminum (left) and zinc (right) powder samples. The symbols are the measured data, averaged over $q$. All $q$-dependence is included in the error bars. 
As an indication as to where the absorption edges occurred in our measurements, we measured absorption spectra with the same sample and same monochromaticity settings (Figure 4). They were recorded with an avalanche photodiode located directly behind the powder samples. The investigated sample areas differed slightly from the ones probed in the speckle patterns. Still, these data show the resolution and performance of the instrument during the speckle experiments.
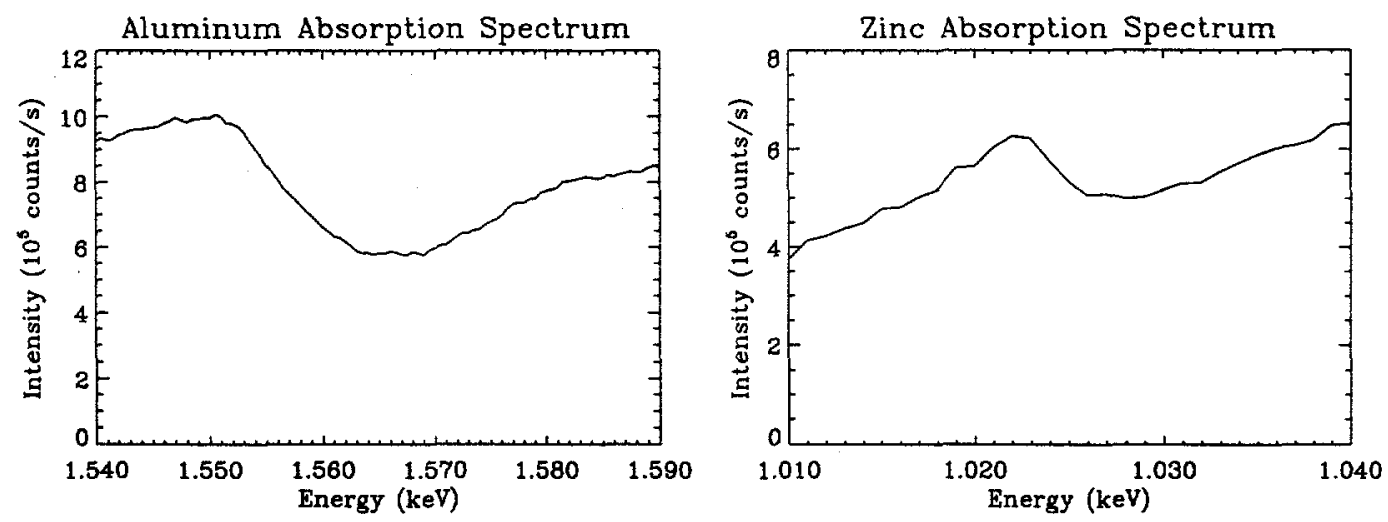

Fig. 4. Absorption spectra taken with the same samples: aluminum powder (left) and zinc powder (right).

\section{Conclusions}

We found a significant speckle contrast variation across the $\mathrm{K}$ and $\mathrm{L}$ absorption edges in powdered aluminum and zinc samples, contrary to common predictions [4,5]. The contrast is decreased at the absorption edge, implying the assumption that $\langle I(k)\rangle$ does not vary significantly over the width of the spectral response function is no longer valid near an absorption edge. We therefore expect that the shape of this variation strongly depends on the monochromator spectral response function and the spatially averaged intensity transmitted by the sample. Our analysis of these contributions to the observed effects is in progress.

This work is supported by the U.S. Department of Energy, Basic Energy Sciences, Office of Science, under contract W-31-109-ENG-38.

\section{References}

[1] M. Sutton et al., "Observation of Speckle by Diffraction with Coherent X-Rays," Nature 352, 608-610 (1991).

[2] Z.H. Cai et al., "Observation of X-ray Speckle by Coherent Scattering at Grazing Incidence," Phys. Rev. Lett. 73, 82-85 (1994).

[3] A.R. Sandy et al., "Production and Characterization of X-ray Speckle at Sector 8 of the Advanced Photon Source," J. Synchrotron Radiat. 6,1174-1184 (1999).

[4] G. Parry, "Speckle Patterns in Partially Coherent Light" in Laser Speckle and Related Phenomena, J.C. Dainty, ed. (Springer-Verlag, Berlin, 1984).

[5] D.L. Abernathy et al., "Small-angle scattering using coherent undulator radiation at the ESRF," J. Synchrotron Radiat., 5, 37 (1998).

[6] I. McNulty et al., "A beamline for $1-4 \mathrm{keV}$ microscopy and coherence experiments at the Advanced Photon Source," Rev. Sci. Instrum., 67

(9), CD-ROM (Sep 1996).

[7] I. McNulty et al., "Multilayer spherical grating monochromator for $1-4 \mathrm{keV}$ x-rays," Proc. SPIE 3150, 195 (1997). 